\title{
Diagnostic pathology: lymph nodes and spleen with extranodal lymphomas
}

\author{
LJ Medeiros, RN Miranda, SA Wang, F Vega, T Muzzafar, \\ CC Yin, CE Bueso-Ramos, P Lin. 2011, \\ Amirsys, Canada
}

Han van Krieken

Published online: 8 September 2011

(C) Springer-Verlag 2011

This book is one of a series with the same format and will consist of 15 volumes. The format is derived from a similar series created by the same publisher for radiology and is new in the pathology field. It is really a reference work in which each lesion is described as follows: title with two typical H\&E stains, low and higher power after which sections are present that consist of a heading with bullet points: terminology, etiology/pathogenesis; clinical issues; macroscopic features; microscopic pathology; ancillary tests; differential diagnosis, and selected references. Furthermore, there is a table with key facts and there are many images, divided in microscopic features and differential diagnosis. Many entities have about two pages of text, but some up to ten. There are 15 chapters, varying from reactive nonspecific changes, to infectious causes of lymphadenitis to the various groups of lymphomas, non-hematopoietic proliferations in lymph nodes, spleen, and an antibody index. The book has, in addition, a website which is regularly updated. The plentiful images are of good quality and indeed most often illustrate what one is looking for; although being trained in the Kiel school with a lot of emphasis on cytological detail, I would often like a somewhat larger magnification and a bit brighter print.

As said, a real reference work which has no sentences but bullet points is not a book to read but to use. I have it now next to my microscope, together with the more typical textbooks and easy access to PubMed and find that

H. van Krieken $(\square)$

Department of Pathology,

Radboud University Nijmegen Medical Centre,

P.O. Box 9101, 6500 Nijmegen, The Netherlands

e-mail: J.vankrieken@pathol.umcn.nl
I use all these tools for different reasons. The "Medeiros" I find the one I most often start with, to check if my memory on a specific feature is correct, to see if an unusual staining in a typical case is really unusual, to go briefly over some differential diagnosis, and to look for maybe further antibodies to use. It is remarkable how much one can find! I can hardly understand how this team from MD Anderson in Houston was able to get all this together so complete and up to date, with references up to 2010. The book is therefore a very useful tool in the practice of a hematopathologist, who, after having reviewed a slide more or less knows which diagnosis is likely, and thus is able to go to the right section of the book. At first glance, my residents like the book too, since it gives a good grip on the criteria that are needed to make a certain diagnosis, and the panel of antibodies they might like to order. However, after they took it to their own office, they realized that it was often difficult to know where to look.

Crucial for books like this one is the index, which is exhaustive. A nice example is the term Carcinoma after which a whole series of lymphoid lesions can be found that are potential mimickers of a carcinoma. Also, one can find entities with different names, indeed at different places, something quite important in lymphoma diagnosis. For instance, there is an entry for both lymphoblastic B-cell lymphoma and for precursor lymphoblastic lymphoma. A pity though, that if one goes for the latter one only gets referred to the former where the page number is given. This is a common nuisance of textbooks. To me it seems simple to add to the reference to the "better" term also the page number.

A special word regarding the chapter on spleen. This chapter covers the lymphoid lesions very well, but not the 
other pathology, like the different forms of hemangioma. This is a bit of a pity because especially on spleen pathology books are scant.

The competition for this book is not so much with another book, but rather with the internet. I am not sure how much of this has to do with age and being brought up and trained with books rather than bits, but my personal preference is the book; much faster and organized than any search machine for the specific purpose of using while reading cases. Of course, in several instances, one likes to get deeper into the matter and go to original articles, but oftentimes the information in the "Medeiros" is sufficient to make a diagnosis with confidence.

In conclusion, a book I recommend, even though it is not nice to read (the only sentences are in the legends of the figures), is quite heavy, and the cover is not really catchy. Pathologists with at least some experience in diagnosing lymph nodes, spleen, and the extranodal lymphoid tissues will find this book a real helpful tool in daily practice, which is, I think, what it was made for. 